Artigos - Gestão do Turismo

\title{
Indicadores de desempenho utilizados pelas empresas hoteleiras da cidade de Braga/PT à luz do balanced scorecard
}

\author{
Performance indicators used by hotel companies in the city of Braga / PT in \\ the light of the balanced scorecard
}

\author{
Indicadores de desempeño utilizados por las empresas hoteleras en la \\ ciudad de Braga / PT a la luz del cuadro de mando integral
}

\author{
Marcleide Maria Macêdo Pederneiras ${ }^{1}$ Robson do Vale Silva ${ }^{1}$ Paula Dutra Leão de Menezes ${ }^{1}$ Jonathan Muniz \\ Soares $^{1}$
}

1 Universidade Federal da Paraíba (UFPB), João Pessoa, PB, Brasil.

Palavras-chave:

Empresas hoteleiras; Indicadores de Desempenho; Balanced Scorecard.

Keywords:

Hotel enterprises: Performance Indicators: Balanced Scorecard.

Palabras clave:

Empresas hoteleras: Indicadores de desempeño: Balanced Scorecard.

\section{Resumo}

A pesquisa objetivou conhecer e analisar os indicadores de desempenho utilizados pelos empreendimentos hoteleiros, da cidade de Braga/PT, evidenciando como são utilizados, à luz da teoria dos indicadores do Balanced Scorecard. Realizou-se uma pesquisa bibliográfica, com a finalidade de levar o conhecimento que já foi explorado na literatura especializada e também uma pesquisa de campo, através da aplicação de um questionário junto aos gestores das empresas hoteleiras enviado por correio eletrônico com o link do questionário. A amostra foi constituída pelas empresas hoteleiras na categoria de 2, 3, 4 e 5 estrelas totalizando 21 hotéis. Os dados foram compilados e tabulados na planilha EXCEL e convertidos em um arquivo compatível com o pacote estatístico Statistical Package for the Social Sciences (SPSS). Concluiu-se que $81 \%$ dos hotéis respondentes utilizam Sistema de Medição de Desempenho (SMD) e que parte destes $(56,1 \%)$ faz a utilização de um sistema de desempenho com indicadores calculados através do EXCEL e em todas as empresas hoteleiras pesquisadas são realizadas reuniões com o objetivo de debater sobre o desempenho. Os indicadores mais utilizados são os financeiros (rentabilidade, receita total e faturamento - valor) e nos indicadores não financeiros, os mais utilizados são os de satisfação dos clientes e fidelidade aos clientes.

Abstract

This research aimed to know and analyse the performance indicators used by hotel enterprises in the city of Braga/PT, identifying how they are used in light of the Balanced Scorecard indicator. A bibliographic research was carried out, with the purpose of bringing to light what has already been explored in specialized literature and a field survey was also carried out, which used as a data collection tool a questionnaire applied to hotel managers sent by e-mail, which included the presentation of the survey, the invitation to participate and the link to the questionnaire. The sample consisted of the hotel companies in the category of 2, 3, 4 and 5 stars totaling 21 hotels. The data was compiled and tabulated in the EXCEL spreadsheet and converted into an archive compatible with the Statistica Package for the Social Sciences (SPSS). It was concluded that $81 \%$ of the responding hotels use a Performance Measurement System (PMS) and that part of these (56.1\%) uses a performance system with indicators calculated through EXCEL and in all the hotel companies surveyed meetings are held to discuss performance. The most used indicators are the financial ones (profitability, total revenue and billing - value) and in the non-financial indicators, the most used are the ones of customer satisfaction and loyalty to customers.

Resumen

La presente investigación tuvo como objetivo conocer y analizar los indicadores de desempeño utilizados por las empresas hoteleras en la ciudad de Braga / PT, identificando cómo se utilizan, a la luz de la teoría de los indicadores del Balanced Scorecard. Se realizó una investigación bibliográfica, 
Revisado por pares.

Recebido em: 18/12/2020.

Aprovado em: 14/09/2021.

Editor:

Glauber Eduardo de Oliveira Santos.

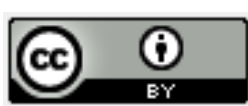

con el propósito de acercar al conocimiento lo ya explorado en la literatura especializada y también se realizó una investigación de campo, que utilizó como instrumento de recolección de datos un cuestionario aplicado a los gerentes de las empresas hoteleras enviado por correo electrónico, que incluía la presentación de la investigación, la invitación a participar y el enlace al cuestionario. La muestra estuvo constituída por empresas hoteleras en la categoría de 2, 3, 4 y 5 estrellas, totalizando 21 hoteles. Los datos fueron compilados y tabulados en la hoja de cálculo EXCEL y convertidos en un archivo compatible con el paquete estadístico Statistical Package for the Social Sciences (SPSS). Se concluyó que el $81 \%$ de los hoteles encuestados utilizan Sistema de Medición del Rendimiento (SMR) y que parte de ellos $(56,1 \%)$ utilizan un sistema de desempeño con indicadores calculados a través de EXCEL y en todas las empresas hoteleras encuestadas se llevan a cabo reuniones para discutir el desempeño. Los indicadores más utilizados son los financieros (rentabilidad, ingresos totales y facturación - valor) y en los indicadores no financieros los más utilizados son los de satisfacción del cliente y la fidelización del cliente.

Como citar: Pederneiras, M. M. M.; Silva, R. V.; Menezes, P. D. L.; Soares, J. M. (2022). Indicadores de desempenho utilizados pelas empresas hoteleiras da cidade de Braga/PT à luz do balanced scorecard. Revista Brasileira de Pesquisa em Turismo, São Paulo, 16, e-2331.

http://doi.org/10.7784/rbtur.v16.2331

\section{INTRODUÇÃO}

Relatório divulgado pela Organização Mundial do Turismo (OMT, 2019) aponta que a movimentação turística mundial cresceu 4\% em 2019. Embora os resultados da movimentação turística tenham sidos positivos em 2019, o crescimento foi mais lento quando comparado as taxas de 2017 (+7\%) e 2018 (+6\%). A incerteza em torno do BREXIT, as tensões geopolíticas e comerciais e a desaceleração econômica global foram os principais fatores que contribuíram para a desaceleração deste crescimento. 0 ano de 2019 foi também o ano de mudanças no setor turístico com a falência da agência de viagens britânica Thomas Cook e de linhas aéreas de baixo custo.

Além disso, a Covid-19 que teve início no final de 2019 na China e se espalhou rapidamente pelo mundo a partir de março de 2020 - quando foi decretado o estado de pandemia pela Organização Mundial da Saúde (OMS) -, ocasionou um grande impacto na economia mundial, incluindo a atividade turística.

A OMT registrou em 2020 uma redução de 74\% na chegada internacionais de turistas em comparação com 2019. Infelizmente, em janeiro de 2021, não houve melhora de desempenho do setor. Segundo a OMT (2021), o indicador da queda foi de $87 \%$ em comparação ao mesmo período do ano anterior, salientando que, com os novos surtos de Covid-19 no início do ano, os países aumentaram as restrições de viagens como contramedida. A região ÁsiaPacífico apresentou queda de 96\%, por ser o local do mundo que continua a ter o maior nível de restrições de viagens em vigor, registrando a maior redução nas chegadas internacionais em janeiro. Europa e África registraram queda de $85 \%$ nas chegadas, enquanto o Oriente Médio apresentou queda de $84 \%$. As chegadas internacionais nas Américas diminuíram $77 \%$ em janeiro.

Olhando para o futuro, a OMT traçou dois cenários para 2021. Os cenários consideram uma série de fatores, como uma melhoria gradual da situação epidemiológica, uma implantação contínua da vacina contra a Covid-19, uma melhoria significativa na confiança do viajante e um grande levantamento das restrições de viagem, em particular na Europa e nas Américas.

O primeiro cenário aponta para uma recuperação em julho, o que resultaria em um aumento de $66 \%$ nas chegadas internacionais para o ano de 2021 em comparação com as mínimas históricas de 2020. Nesse caso, as chegadas estariam ainda $55 \%$ abaixo dos níveis registrados em 2019. 0 segundo cenário considera uma potencial retomada em setembro, levando a um aumento de $22 \%$ nas chegadas em relação ao ano passado. Ainda assim, seria $67 \%$ abaixo dos níveis de 2019.

Contudo, destaca-se que o setor turístico e, especificamente de meios de hospedagem, mais especificamente de meios de hospedagem, é muito sensível às consequências da pandemia, o que foi mostrado pela queda expressiva das atividades nesse setor. Gullo (2020) analisou os impactos na economia, sobretudo, de acordo com o INE (2020), utilizando o sistema de matrizes Simétricas de Input- Output, que é um dos instrumentos analíticos que prevê o impacto da pandemia Covid-19 na economia portuguesa. O INE menciona que foi simulado o efeito de uma redução anual da atividade turística de $25 \%$, seja do turismo de visitantes não residentes quer seja do turismo interno, traduzindo-se numa redução de $2,9 \%$ do PIB anual em Portugal.

A pesquisa foi realizada na cidade de Braga, localizada na região do Minho/PT, que se destaca no turismo. Em dados totais, em agosto de 2017, a cidade de Braga recebeu 325 mil visitantes. "Com esta evolução, comparando agosto de 2017 e agosto de 2018, houve um crescimento de mais de 10 mil pessoas, um crescimento de mais de 
5\%", referiu o vereador do Município de Braga, Altino Bessa, durante evento de apresentação dos dados do turismo em Braga, no âmbito da comemoração do dia Mundial do Turismo.

A procura turística em Braga tem sofrido um crescimento significativo nos últimos anos, sendo que "se contabilizarmos o número de visitantes entre 2013 e 2018 chegamos a 1 milhão e 500 mil turistas a entrar neste equipamento municipal". Esse relato evidencia o crescimento turístico nessa cidade e a importância de se estudar como os hotéis utilizam os medidores de desempenho dos empreendimentos que dão suporte à atividade turística.

Vale registrar que o todo setor hoteleiro está envolvido em um ambiente competitivo (Callado; Almeida; Callado, 2008), em que se faz necessário obter informações adequadas para a gestão das atividades (Haktanir \& Harris, 2005). 0 surgimento de novas empresas hoteleiras ou a manutenção das já existentes demandam de seus gestores o desenvolvimento e a implantação de atividades estratégicas que coloquem à disposição dessas organizações meios para a obtenção de um perfil que Ihes assegurem a continuidade. Nesse contexto, tem-se como alguns elementos do mercado atual a concorrência e as mudanças econômicas. Como consequência, as empresas hoteleiras devem buscar vantagens competitivas para continuarem atuantes nesse ambiente dinâmico e instável marcado pela sazonalidade que constitui uma característica do setor.

Isto posto, faz-se necessário instituir medidas ou indicadores de desempenho com o propósito de mensurar o comportamento das empresas e obter informações para a tomada de decisões, a fim de que essas se mantenham no mercado.

Esses indicadores são ferramentas centrais as quais possibilitam um acompanhamento das mais importantes opções de interesse da empresa e, por isso, permitem o planejamento de ações que objetivam melhorias de desempenho organizacional e financeiro (Rodrigues, Schuch, \& Pantaleão, 2003).

Quesado e Rodrigues (2009, p. 96) afirmam que:

dadas as limitações dos indicadores de gestão tradicionais para avaliar o desempenho das empresas, centrados essencialmente em indicadores financeiros orientados para o curto prazo, torna-se necessário o desenvolvimento de modelos de indicadores de gestão que traduzam todos os fatores críticos de sucesso.

Entretanto, esses indicadores financeiros, para Kaplan e Norton (1997), não são suficientes para nortear e analisar a trajetória da empresa em um ambiente competitivo, pois contam apenas parte da história das ações passadas e não fornecem informações adequadas para as ações do presente e do futuro, que devem ser concretizadas para definir o valor financeiro. Apesar dos estudiosos do assunto criticarem fortemente esses indicadores, os mesmos, quando da avaliação de resultados e desempenhos, devem ser utilizados, pois são provas concretas dos efeitos de todas as outras medidas.

Para Banker, Poter e Srinivasan (2000), os indicadores não-financeiros são mais eficazes para o futuro que os financeiros, auxiliando os administradores a focarem suas ações nas perspectivas a longo prazo.

De acordo com pesquisa realizada pelos membros do American Institute of Public Accountants (AICP) (Maisel, 2001), 43\% utilizavam a técnica do BSC apresentada em quatro trabalhos de Kaplan e Norton (1992, 1993, 1996a, b) e em maior detalhe em dois livros dos mesmos autores (Kaplan \& Norton, 1996 a, b, c, 2001). Trabalhos publicados posteriormente, como Hoffecker e Goldenberg (1994), Lingle e Schiemann (1996), Kutzman (1997) e Frigo e Krumwied (2000) propagaram a divulgação das características da técnica do BSC e deram ênfase de um sistema de medição de desempenho para um sistema de gerenciamento e implementação de estratégia.

Alguns estudos empíricos mais recentes como os de Braam e Nijssen (2011), Kollberg e Elg (2011), Bisbe e Barrubés (2012), Hendricks et al. (2012), Machado (2013), Greiling (2010), demonstraram resultados diversos que serão tratados posteriormente.

Quesado e Rodrigues (2009, p. 96) ressaltam que o Balanced Scorecard surgiu como uma importante ferramenta de Contabilidade de Gestão Estratégica, capaz de "refletir os fatores críticos de sucesso e um conjunto de medidas financeiras e não-financeiras estruturadas em quatro perspectivas básicas", que de acordo com Kaplan e Norton (1997) são: financeira; clientes; processos internos; e aprendizagem e crescimento, perspectivas essas que serão tratadas posteriormente.

Assim, considerando a importância dos indicadores de desempenho, sobretudo em empresas hoteleiras de regiões onde o turismo tem tido um crescimento significativo, como é o caso de Portugal, surge à questão: Quais os indicadores da medição de desempenho utilizados pelas empresas hoteleiras da cidade de Braga/PT e como são usados na melhoria do desempenho dos hotéis? 
Para responder a este problema e considerando que há vários modelos ou sistemas de medição de desempenho, esta pesquisa objetiva conhecer e analisar os indicadores da medição de desempenho utilizados pelas empresas hoteleiras da cidade de Braga/PT e como são usados na melhoria do desempenho dos hotéis nas perspectivas teóricas do Balanced Scorecard.

Para tanto, ao longo do estudo identificou-se como é realizado o processamento das informações dos sistemas de medição de desempenho das empresas hoteleiras e os indicadores utilizados, bem como, as características dos sistemas de controle e avaliação do desempenho organizacional e a utilização na tomada de decisões das empresas hoteleiras e demonstrando a utilização dos indicadores na perspectiva do BSC.

Neste panorama, com tanta diversidade no turismo cultural e religioso, como é o caso da cidade de Braga/PT, esse trabalho se justifica.

Justifica-se ainda, considerando que o estudo dos indicadores utilizados nos hotéis de Braga-Portugal, trará com seus resultados, benefícios potenciais para a área de administração, contabilidade, turismo e hotelaria e para a sociedade/academia portuguesa e também brasileira, viabilizando, sobremaneira aos gestores dos hotéis uma ressignificação no uso de novos indicadores de medição de desempenho até então não utilizados nas empresas hoteleiras brasileiras. Ou seja, pretende-se, a partir do estudo realizado, caracterizar um conjunto de contribuições para a formação de um perfil inovador dos profissionais e dos futuros profissionais na área da gestão hoteleira de forma qualificada.

\section{MEDIÇÃO E SISTEMAS DE DESEMPENHO ORGANIZACIONAL}

Na concepção de Atkinson et al. (2000, p. 36) a Contabilidade Gerencial é "um processo de identificar, mensurar, reportar e analisar informações sobre os eventos econômicos da empresa". Para Crepaldi $(2008$, p. 5) a Contabilidade busca coletar, apresentar e interpretar os fatos econômicos. Na visão de Nascimento et al. (2015, p. 6), a Contabilidade Gerencial "existe devido a necessidade de informações que surgem diariamente nas organizações, sejam elas micro, pequenas, médias ou empresas de grande porte." Os autores completam assegurando que não é aceitável que uma organização sobreviva por muito tempo sem o mínimo de conhecimento sobre o que acontece internamente.

Atualmente, percebe-se que qualquer tipo de organização precisa de informações que sustentem sua continuidade e promovam sua competitividade, apesar dos obstáculos mercadológicos. Nesse sentido, as informações gerenciais são indispensáveis. As empresas hoteleiras têm a característica da sazonalidade, o que faz surgir a necessidade de que seus gestores planejem suas atividades empresariais de forma estratégica no curto, médio e longo prazos, levando em consideração essa característica do negócio.

Os gestores das organizações devem se ater às questões que envolvam o desempenho e a permanência das organizações no mercado e, para tanto, necessitam de informações úteis ao processo de tomada de decisão, notadamente em cenários econômico-políticos de mudanças e de acirrada competitividade. Crepaldi (2008, p.2) corrobora quando afirma que "as empresas estão em constantes mudanças e cada vez mais necessitam de controles precisos e de informações oportunas sobre seu negócio para adequar suas operações às novas situações de mercado."

Assim, destaca-se uma das características mais marcantes da medição e avaliação de desempenho: o controle. Os controles gerenciais e operacionais permitem que a organização avalie se as metas traçadas estão sendo alcançadas, permitindo a construção de novos modelos estratégicos voltados à manutenção ou adaptação das metas organizacionais. Na percepção de Atkinson et al. (2000, p.94), controle é o conjunto de métodos e instrumentos que os membros da empresa usam para mantê-la em acordo com os planos traçados, a fim de obter seus objetivos.

Nesse contexto, é oportuno definir o que é desempenho. Lebas e Euske (2002, citado por Barbosa, 2010, p.9) o definem da seguinte forma:

é a soma de todos os processos que levarão os administradores a adotarem ações apropriadas no presente que criarão uma organização com desempenho no futuro, ou seja, fazer hoje o que levará ao aumento do valor amanhã.

Como também pode ser compreendido como "o ato de medir o conjunto de atividades, pressupostos e técnicas que visam a quantificar variáveis e atributos de interesse do objeto a ser analisado” (Kiyan, 2001, p.25). 
Dessa forma, quando a organização aloca todas as medidas utilizadas para quantificar a eficiência e a eficácia de suas ações, tem-se um sistema de medição de desempenho.

Como referenda Lugoboni et al. (2013), os modelos de avaliação de desempenho organizacional aparecem como principais métodos para mensurar o controle gerencial e seu alinhamento estratégico. Corrobora, ainda, Crispim e Lugoboni (2012), quando afirmam que as medidas de desempenho sofrem influências pelas perspectivas da organização, sendo elas a estrutura organizacional, o sistema de recompensas e o ambiente operacional. Os autores citados apontam os principais modelos de avaliação de desempenho: Painel de Controle de Bordo (Tableau de Bord); Administração por Objetivos (APO); Método das Áreas Chaves de Sucesso; Método de Buchele; Método da Avaliação Global de Desempenho de Corrêa; The Balanced Scorecard (BSC); Performance Measure Questionnaire (PMQ); Strategic Measuremente and Reporting Techinique (SMART); Sete Critérios de Desempenho (SCD); Modelo Quantum de Medição de Desempenho (MQDM); Método de Rummler e Branche; Integral and Dynamic Performance Measurement System (IDPMSB); Skandia Navigator; Sigma Sustainability Scorecard; Value Explorer (VE); Value Chain Scoreboards (VCS ); Performance Prism (PP); e Prêmio Nacional de Qualidade (PNQ).

\section{BALANCED SCORECARD (BSC)}

De acordo com Kaplan e Norton (1997), o Balanced Scorecard é uma técnica que visa integrar e equilibrar todos os principais indicadores de desempenho existentes em uma empresa, desde financeiros e administrativos até aqueles relacionados aos processos internos, estabelecendo objetivos de qualidade (indicadores) para funções e níveis fundamentais dentro da organização. Isto é, a incrementação dos indicadores corporativos em setores, com metas claramente estabelecidas. Assim, este modelo traduz a missão e estratégia de uma empresa em objetivos e medidas tangíveis. As medidas representam o equilíbrio entre os variados indicadores externos (com foco em acionistas e clientes) e as medidas internas de processos críticos de negócios (como aprendizado, crescimento e inovação).

Dessa forma, o BSC possui quatro perspectivas, sendo elas:

- $\quad$ Financeira - aborda indicadores que demonstrem a saúde financeira do negócio.

- $\quad$ Clientes - são os indicadores relacionados à satisfação dos clientes.

- $\quad$ Processos Internos - trata da forma pela qual os processos internos agregam valor ao cliente.

- Aprendizado e do Crescimento - analisa a capacidade da organização aprender com as suas experiências e utilizá-las para crescer e avançar.

Unidas, compõem um conjunto harmônico e interdependente com seus "objetivos e indicadores se interrelacionando e formando um fluxo ou diagrama de causa e efeito que se inicia na perspectiva do aprendizado e crescimento e termina na perspectiva financeira" (Faria, 2007, p. 19).

Segundo Kaplan e Norton (1992), o BSC descreve a visão como "para onde iremos" e a estratégia seria "como iremos" no centro do processo de gestão, estabelecendo objetivos e assumindo que as pessoas deverão adotar determinados comportamentos e ações que se tornam necessárias para atingi-los. O BSC, não tem por objetivo a definição da estratégia da empresa, mas sim a sua sistematização para a construção do mapa estratégico, com o objetivo de realizar o acompanhamento e a monitorização dos resultados e da aprendizagem da organização.

O mapa estratégico esboça a todos os colaboradores, de forma a poderem contribuir para a realização dos objetivos e a saberem como devem atuar. A falta de participação dos colaboradores com os objetivos estratégicos será o primeiro sinal de desalinhamento e quebra da performance estratégica. E, por fim, o BSC pressupõe um acompanhamento periódico à execução da estratégia, permitindo no curto prazo que a organização possa comparar a sua performance com as metas definidas.

\section{INDICADORES DE DESEMPENHO ORGANIZACIONAIS EM EMPRESAS HOTELEIRAS}

Como já mencionado anteriormente, todos os tipos de organizações, independentemente do porte ou do ramo de negócios, necessitam de parâmetros de medição e avaliação de desempenho. No que se refere a hotéis, Lunkes (2004) diz que um efetivo controle gerencial baseado em sistemas de medição de desempenho é capaz de promover melhorias gradativas no desempenho e na competitividade de empresas hoteleiras. 
Lunkes (2004) expõe algumas medidas que podem ser usadas para comparar o desempenho em hotéis, como: competência dos colaboradores e sua motivação; pessoal qualificado para realizar mudanças e inovações; inovações tecnológicas, de produtos ou organizacionais; o hotel deve ser eficiente no tempo do ciclo entre o início e o término de um processo, que pode incluir a correção de erros a qualquer momento; percepção em relação ao atendimento antes, durante e depois da hospedagem, etc.

Os principais indicadores de desempenho que são utilizados pelo setor hoteleiro, descritos por Lugoboni, Fontes, Andrade (2013, p. 8-9), referenciados na literatura por vários autores e agrupados em dimensões são: na Dimensão econômica-financeira a Evolução da lucratividade; o Fluxo de caixa; a Margem de lucro; a Rentabilidade; a Receita total; o Retorno sobre o investimento; o Valor Econômico Agregado (EVA); a Diária Média Planas; o Valor de Mercado Agregado (MVA); a Liquidez geral; a RevPAR Planas; a Dimensão operações a Taxa de ocupação; a Evolução no número de clientes; a Porcentagem de metas atendidas; o Índice de adequação microbiológica (análise dos alimentos); na Dimensão stakeholders o posicionamento no segmento; o Número de clientes fidelizados e; o Nível de satisfação.

Segundo Merchant (2006), acredita-se que a construção de indicadores de desempenho de forma adequada está diretamente relacionada ao sucesso de um sistema de avaliação de desempenho organizacional. Desse modo, algumas características, critérios e propriedades são fundamentais, a fim de que sejam percebidas no momento da construção desses indicadores, pois os indicadores devem ser acurados e resumem-se em precisão e objetividade; devem ser compreensíveis, o gestor deve conhecer o que está medindo; e devem ter uma relação que compense custo $x$ benefício, para isso, o custo para realizar a medição tem de ser menor do que os benefícios trazidos pelo indicador de seu monitoramento.

Os autores Bortoluzzi, Ensslin e Ensslin (2010) chegam a um resultado, em que um indicador de desempenho é mensurável quando representa a percepção de valor que o gestor responsável deseja monitorar e não a medida mais fácil associada ao nome do indicador de desempenho. Seria operacional, quando fisicamente passível de mensurar a propriedade a ele associada e inteligivel quando todos tiverem o mesmo entendimento de seus níveis, podendo o fácil entendimento estar associado a não ambiguidade e a redundância. É homogêneo quando todos os seus niveis analisarem as possiveis ocorrências das mesmas propriedades do contexto, permitindo distinguir o que falta para melhorar quando em todos os seus niveis é possível visualizar, nas propriedades do contexto que mensura, qual o desempenho pior e qual o melhor, e por fim, um indicador de desempenho respeita as propriedades das escalas quando evidencia de forma exaustiva quais seus possíveis níveis, a ordenação desses níveis e a diferença de atratividade entre eles.

Por isso, considerando as características dos indicadores de desempenho, os gestores devem ter cuidado na construção dos que irão utilizar.

\section{CENÁRIO ATUAL}

\subsection{Prestação de serviços na hotelaria}

A hotelaria é uma das atividades essenciais dentro do sistema turístico, visto que para o turismo acontecer, independente do segmento, a hotelaria deve estar presente (Mota, 2001). 0 setor de serviços, no qual os hotéis estão inseridos, oferece uma variedade de atividades. Dessa forma, a indústria hoteleira apresenta inúmeras tarefas que exigem certa qualificação (Popp et al., 2007).

Considerando que é uma atividade de prestação de serviços, apresentando características próprias de organização, tem como objetivo principal oferecer hospedagem, alimentação (a depender do tipo de hospedagem), segurança, entre outros, ligados à atividade do bem receber do turista. É preciso que o estabelecimento atenda às necessidades básicas dos hóspedes, satisfazendo e/ou superando suas expectativas (Badaró, 2010).

Algumas das características principais da atividade hoteleiro de acordo com Petrocchi (2003) são: intangibilidade, impossibilidade de estocagem do serviço, produção e consumo fisicamente unidos, influências externas e sazonalidade. Tais características diferenciam-se da administração tradicional da indústria de produtos. Nesse sentido, Barros e Badiz (2005, p. 1001) contribui com esta afirmação ao explicar que "a tarefa de administrar não é simples, especialmente quando se trata de um empreendimento tão diversificado quanto um hotel, e, ainda, por se tratar de uma indústria de serviços, cujas diferenças são marcantes em relação à indústria de bens". Assim sendo, as maneiras de lidar com os desafios podem ser diferentes da gestão de outros tipos de administração. 
Paiva et al. (2010, p.10) expõe que "para o setor hoteleiro, quanto maior a consciência dos envolvidos na prestação de serviços, na busca pela qualidade da excelência, maior é o diferencial perante seus concorrentes". Portanto, a qualidade do serviço prestado na hotelaria estabelece uma questão de sobrevivência empresarial. Atualmente, o profissional do setor de serviços, em especial o de hotelaria, precisa sempre estar acompanhando as mudanças de perfil dos hóspedes (Barros \& Badiz, 2005).

\subsection{Cidade de Braga/PT}

A cidade de Braga, localizada na região do Minho/PT, possui um vasto patrimônio cultural, cuja maior representação é o Santuário do Bom Jesus do Monte. Patrimônio Mundial da UNESCO recebeu o título de Cidade Europeia do Desporto e Cidade Criativa da UNESCO, na categoria Media Arts. Em 2019, foi eleita o segundo Melhor Destino Europeu do Ano segundo Desporto (2018). De acordo com dados disponíveis no site semanariov.pt (2018), em dados totais, em agosto de 2017 a cidade de Braga recebeu 325 mil visitantes e "com esta evolução, comparando agosto de 2017 e agosto de 2018, houve um crescimento de mais de 10 mil pessoas, um crescimento de mais de $5 \%$ ", referiu o vereador do Município de Braga, Altino Bessa, durante evento de apresentação dos dados do turismo em Braga, no âmbito da comemoração do dia Mundial do Turismo.

Ainda de acordo com o mesmo site (2018), a procura turística em Braga tem sofrido um crescimento significativo nos últimos anos, sendo que "se contabilizarmos o número de visitantes entre 2013 e 2018, chegamos a 1 milhão e 500 mil turistas a entrar neste equipamento municipal”.

A matéria conclui que este crescimento turístico, segundo indicadores, levou à criação de novas unidades de alojamento local. Em 2020 foram pedidas 121 licenças para esse tipo de alojamento. "Em 2010 tínhamos 1 alojamento local, em 2013 tínhamos 4 alojamentos locais e em 2018 temos 278 alojamentos locais", segundo dados lançados por Altino Bessa (2018), adiantando que "com este total de 278 alojamentos locais, temos uma capacidade de cerca de 813 quartos com a possibilidade de acolher 1774 turistas".

Podemos notar que, no início de 2020, o turismo estava em alta e o cenário era muito animador, infelizmente com a propagação do vírus Covid-19 o cenário mudou, o secretário-geral da OMT, Zurab Pololikashvili (2020) disse que: "O mundo enfrenta uma crise sanitária e econômica sem precedentes, o turismo foi duramente atingido, com milhões de empregos em risco".

De acordo com o INE (2020) utilizando o sistema de matrizes Simétricas de Input- Output que é um dos instrumentos analíticos que prevê o impacto da pandemia Covid-19 na economia portuguesa, o INE menciona que foi simulado o efeito de uma redução anual da atividade turística de $25 \%$, seja do turismo de visitantes não residentes quer seja do turismo interno, traduzindo-se numa redução de 2,9\% do PIB anual em Portugal.

Em junho de 2021, foram tomadas medidas para recuperar a livre circulação na União Europeia (UE) e eliminar as restrições ao turismo, setor mais afetado economicamente pela pandemia de coronavírus. Estes são os principais objetivos do Certificado Digital Covid-19 da UE que começa a ser emitido em Portugal.

Após ser aprovado pelo Parlamento Europeu, o regulamento foi assinado na última segunda-feira, em Bruxelas, pela presidente da Comissão Europeia, Ursula von der Leyen, e pelo primeiro-ministro português, presidente rotativo do Conselho da UE, António Costa. (AMATO, 2021).

\section{METODOLOGIA}

Esta investigação, quanto aos fins, é caracterizada como exploratória - uma vez que se propõe a compreender e ampliar o conhecimento sobre indicadores de desempenho e o processo de tomada de decisão no setor hoteleiro. E descritiva porque se buscou identificar os indicadores de desempenho utilizados pelas empresas hoteleiras pesquisadas, assim como se confrontou os achados da prática do setor hoteleiro com a literatura do referido estudo.

No que se refere ao método abordado no estudo, classifica-se como dedutivo, visto que a pesquisa realizou-se a partir de dados obtidos junto a gestores de empresas do ramo da hotelaria, tentando generalizar os resultados da amostra para a população.

Quanto aos meios, esta pesquisa é bibliográfica e de campo, pois se desenvolve no âmbito dos hotéis, localizados em Portugal na cidade de Braga. 
O universo da pesquisa constitui-se das empresas do ramo da hotelaria na categoria de 2 a 5 estrelas. De acordo com as informações contidas no site business.turismodeportugalpt, o processo de classificação dos empreendimentos turísticos em Portugal destina-se a atribuir, confirmar ou alterar a tipologia, o grupo (quando aplicável) e a categoria dos empreendimentos turísticos, mediante um conjunto de requisitos que se encontram estipulados, ou genericamente no Regime Jurídico dos Empreendimentos Turísticos (RJET), ou nos regulamentos por tipologias, que dele derivam.

Ainda de acordo com as informações contidas no site business.turismodeportugalpt, os estabelecimentos hoteleiros são classificados nas categorias: Hotéis - 1 a 5 estrelas e Hotéis-Apartamentos - 1 a 5 estrelas. Os requisitos para classificá-los são definidos através da portaria regulamentar que incidem sobre: Características das instalações e equipamentos; Serviço de recepção e portaria; Serviço de limpeza e lavandaria; Serviço de alimentação e bebidas e; Serviços complementares. A referida portaria descreve requisitos mínimos e requisitos opcionais, cujo somatório determina a pontuação necessária para obter determinada classificação.

A presente pesquisa foi realizada com os gestores das empresas hoteleiras da cidade das categorias 2 a 5 estrelas, que, de acordo com dados obtidos em websites, possui 29 (vinte e nove) estabelecimentos hoteleiros. A amostra foi composta por 21 hotéis cujos gestores se dispuseram a responder a investigação a partir de contatos via e-mail.

O instrumento para coleta de dados foi um questionário composto por perguntas abertas e fechadas, com predominância destas últimas, adaptadas à realidade das empresas hoteleiras da região a ser estudada. A escolha da aplicação do questionário foi motivada por este instrumento atender aos objetivos propostos na investigação e pela maior probabilidade de retorno dos questionários respondidos, uma vez que o mesmo foi inserido em plataforma online, a exemplo do Google Forms, ficando disponível para os respondentes, coletando dados necessários ao desenvolvimento do estudo.

Os dados obtidos com a aplicação dos questionários foram organizados em uma planilha EXCEL e convertidos em um arquivo compatível com o pacote estatístico Statistical Package for the Social Sciences (SPSS), de onde foram produzidas as informações em tabelas, gráficos e medidas descritivas (frequência absoluta, frequência percentual, médias, medianas).

Para análise teórica dos indicadores de desempenho coletados das empresas hoteleiras pesquisadas, usaram-se as quatro perspectivas de Kaplan e Norton: financeira, clientes, processos internos e aprendizagem e crescimento.

\section{APRESENTAÇÃO E ANÁLISE DOS RESULTADOS}

\subsection{Perfil dos respondentes}

A partir da Tabela 1, constata-se que o perfil dos respondentes é majoritariamente masculino (60,2\%). Este resultado vem corroborar a predominância da presença masculina na gestão hoteleira. Verifica-se ainda que a maioria (55,9\%) possui grau de instrução licenciatura completa e (10,9\%) superior incompleto. A maioria dos gestores são gerentes $(43,8 \%)$ e proprietários (42,4\%). A minoria são diretores comerciais $(8,2 \%)$ ou financeiros $(5,4 \%)$.

\begin{tabular}{|c|c|c|c|}
\hline Variáveis & Categoria & $\mathbf{N}$ & $\%$ \\
\hline \multirow[t]{4}{*}{ Sexo } & Feminino & 8 & 39,7 \\
\hline & Masculino & 13 & 60,2 \\
\hline & Secundário & 2 & 9,5 \\
\hline & Superior incompleto & 3 & 10,9 \\
\hline \multirow[t]{4}{*}{ Grau de Instrução } & Licenciatura (Bacharelato) & 11 & 55,9 \\
\hline & Mestrado & 4 & 19,0 \\
\hline & Doutoramento & 1 & 4,7 \\
\hline & Proprietário (Sócio) & 9 & 42,4 \\
\hline Posição hierárquica & Diretor financeiro & 1 & 5,4 \\
\hline \multirow[t]{2}{*}{ Na empresa } & Diretor comercial & 2 & 8,2 \\
\hline & Outro (gerente) & 9 & 43,8 \\
\hline
\end{tabular}

Fonte: Elaboração própria (2020).

Constata-se na Tabela 2 que as Licenciaturas mais frequentes são Gestão, Economia e Turismo, totalizando 52,2\%. Apenas 9,5\% dos respondentes não fizeram pós-graduação, dos que realizaram pós-graduação 14,2\% dos gestores 
fizeram mestrado em gestão, sendo 14,2\% na área de hotelaria e 14,2\% em administração, 14,2\% também na área de gestão hoteleira e dois (9,5\%) na área de turismo. Para Leite (2004), um aumento nos cursos de pósgraduação, por exemplo, poderia vir a suprir carências na melhoria gerencial.

Tabela 2 - Perfil do respondente quanto à sua Licenciatura e Pós-graduação

\begin{tabular}{|c|c|c|c|c|c|}
\hline Licenciatura (Bacharelato) & $\mathbf{N}$ & $\%$ & Pós-graduação & $\mathbf{N}$ & $\%$ \\
\hline Não fez & 2 & 9,5 & Não fez & 2 & 9,5 \\
\hline Gestão de Qualidade & 2 & 9,5 & Gestão hoteleira & 3 & 14,2 \\
\hline Contabilidade & 1 & 4,7 & Turismo & 2 & 9,5 \\
\hline Economia & 4 & 19,0 & Economia & 1 & 4,7 \\
\hline Gestão & 4 & 19,0 & Gestão & 3 & 14,2 \\
\hline Turismo & 3 & 14,2 & Adm. em Marketing & 2 & 9,5 \\
\hline Gestão Hoteleira & 1 & 4,7 & Direção em Hotelaria & 1 & 4,7 \\
\hline Administração & 1 & 4,7 & Hotelaria & 3 & 14,2 \\
\hline Engenharia Industrial & 1 & 4,7 & Design e Marketing & 1 & 4,7 \\
\hline Gestão do Turismo & 2 & 9,5 & Administração & 3 & 14,2 \\
\hline TOTAL & 21 & 100,0 & TOTAL & 21 & 100,0 \\
\hline
\end{tabular}

Fonte: Elaboração própria (2020).

\subsection{Informações gerais sobre as empresas hoteleiras}

Pode-se observar na Tabela 3 que 57,1\% dos hotéis são classificados como de duas ou três estrelas. Quanto ao enquadramento dos hotéis, a maioria, correspondendo a 66,6\%, enquadra-se no tipo de hospedagem de lazer, descanso e negócio. Essa abordagem mista tem uma percentagem elevada e este resultado, de acordo com Leite (2004), deve-se ao fato de que durante a alta estação alta os hotéis recebem, na quase totalidade de sua oferta, hóspedes para lazer e descanso, mas na estação baixa lançam pacotes atrativos para a hospedagem de negócios. Desta forma, os hotéis conseguem diminuir os efeitos da sazonalidade. Ainda se constata na Tabela 3 que a maioria, representando $57,1 \%$, não utiliza qualquer financiamento bancário. Esse dado demonstra que são poucos os hotéis de Braga que se encontram endividados, inclusive nenhum usa formas de financiamento factoring.

Tabela 3 - Informações gerais sobre as empresas hoteleiras

\begin{tabular}{llll}
\hline \multicolumn{1}{c}{ Variável } & Categoria & $\mathrm{N}$ & \% \\
\hline & Duas estrelas & 5 & 23,8 \\
& Três estrelas & 7 & 33,3 \\
& Quatro estrelas & 8 & 38,0 \\
& Cinco estrelas & 1 & 4,7 \\
Enquadramento deste hotel & Hosp. Lazer e descanso & 6 & 28,5 \\
& Hospedagem de negócio & 1 & 4,7 \\
Costuma utilizar financiamento de que & 14 & 66,6 \\
tipo? & Hospedagem de lazer, descanso e negócio & 8 & 38,0 \\
& Sim, de instituição financeira & 0 & 0,0 \\
& Sim, de factoring & 1 & 4,7 \\
& Sim, de leasing & 12 & 57,1 \\
\hline
\end{tabular}

Fonte: Elaboração própria (2020).

As informações apresentadas na Tabela 4 apresentam três variáveis, tempo de mercado, unidades habitacionais e funcionários que, em média, são respectivamente 25 anos, 30 unidades habitacionais e 11 funcionários, o que significa que os hotéis são em geral medianos.

Tabela 4 - Informações descritivas gerais sobre as empresas hoteleiras do tempo de mercado, unidades habitacionais e número de funcionários

\begin{tabular}{lrrrr}
\hline \multicolumn{1}{c}{ Variável } & Média & Mediana & Mínimo & \multicolumn{1}{c}{ Máximo } \\
\hline Tempo de Mercado & 25,06 & 20,0 & 2 & 85 \\
Unidades habitacionais & 30,69 & 22,0 & 4 & 113 \\
Funcionários & 11,80 & 10,0 & 2 & 50 \\
\hline
\end{tabular}

Fonte: Elaboração própria (2020). 


\subsection{Processamento das informações dos sistemas de medição de desempenho das empresas hoteleiras e respon- sáveis pela coleta de dados}

Na Tabela 5 evidenciou-se que 80,9\% dos hotéis têm um Sistema de Medição de Desempenho (SMD) e foi recomendado predominantemente por consultores $(76,1 \%)$ ou aprendido pelos próprios gestores $(9,5 \%)$. Estes resultados corroboram com Lugoboni et al. (2014), quando afirmam que os modelos de avaliação de desempenho organizacional aparecem como principais métodos para mensurar o controle gerencial e seu alinhamento estratégico.

Destaca-se, também, a utilização de um sistema de desempenho, majoritariamente, com indicadores calculados através do EXCEL $(56,1 \%)$ e $34,2 \%$ dos hotéis têm software próprio. Estes indicadores são coletados pelo diretor ou gerente $(42,4 \%)$ ou contabilista $(26,0 \%)$.

\begin{tabular}{|c|c|c|c|}
\hline Variável & Categoria & $\mathrm{N}$ & $\%$ \\
\hline \multirow[t]{3}{*}{ Possuem um sistema de medição de desempenho (SMD) do hotel } & Sim & 17 & 80,9 \\
\hline & Não & 4 & 19,0 \\
\hline & Empresa-mãe & 0 & 0,0 \\
\hline \multirow[t]{5}{*}{ Se há sistema de medição quem o recomendou? } & Conhecimento aprendido pelos gestores & 2 & 9,5 \\
\hline & Consultores das áreas & 16 & 76,1 \\
\hline & Não tem & 0 & 0,0 \\
\hline & Outros & 3 & 14,2 \\
\hline & Contabilista & 5 & 26,0 \\
\hline \multirow[t]{3}{*}{ Responsável pela coleta de dados para o SMD do hotel } & Diretor ou gerente & 9 & 42,4 \\
\hline & Funcionário treinado & 5 & 23,2 \\
\hline & Outro & 2 & 8,2 \\
\hline \multirow[t]{3}{*}{ Software específico para calcular os indicadores } & Sim & 7 & 34,2 \\
\hline & Não, usa-se o EXCEL & 12 & 56,1 \\
\hline & Outros & 2 & 9,5 \\
\hline
\end{tabular}

Fonte: Elaboração própria (2020).

\subsection{As características dos sistemas de controle e avaliação do desempenho organizacional}

A Tabela 6 demonstra que 31,5\% dos hotéis possuem certificação de qualidade e apenas 8,2\% estão com certificação meio-ambiental em curso. Constata-se que $45,2 \%$ dos hotéis fazem pesquisa para avaliar a satisfação dos seus funcionários.

Tabela 6 - Características dos sistemas de controle e avaliação do desempenho organizacional

\begin{tabular}{|c|c|c|c|}
\hline Variável & Categoria & $\mathrm{N}$ & $\%$ \\
\hline \multirow[t]{3}{*}{ Certificação de qualidade } & Sim & 7 & 31,5 \\
\hline & Em curso & 1 & 4,1 \\
\hline & Não & 13 & 64,3 \\
\hline \multirow[t]{3}{*}{ Certificação de meio-ambiente } & Sim & 2 & 8,2 \\
\hline & Em curso & 2 & 8,2 \\
\hline & Não & 17 & 83,5 \\
\hline \multirow{6}{*}{$\begin{array}{l}\text { No hotel pesquisa-se sobre a sa- } \\
\text { tisfação dos funcionários } \\
\text { Realiza-se pesquisa para medir a } \\
\text { satisfação dos hóspedes }\end{array}$} & Sim & 9 & 45,2 \\
\hline & Não & 12 & 54,7 \\
\hline & Sim & 19 & 90,4 \\
\hline & Não & 2 & 9,5 \\
\hline & Mensalmente & 4 & 19,0 \\
\hline & Bimestralmente & 0 & 0,0 \\
\hline \multirow{4}{*}{$\begin{array}{l}\text { Frequência da realização da pes- } \\
\text { quisa }\end{array}$} & Trimestralmente & 1 & 4,7 \\
\hline & Semestralmente & 2 & 9,5 \\
\hline & Anualmente & 0 & 0,0 \\
\hline & Outro (diariamente) & 14 & 66,6 \\
\hline \multirow{5}{*}{$\begin{array}{l}\text { Atributos escolhidos pelo gestor } \\
\text { do hotel para identificar o nível de } \\
\text { satisfação dos seus hóspedes* }\end{array}$} & Acesso a informações ... (Booking) & 15 & 71,4 \\
\hline & Estudos próprios para analisar a imagem & 2 & 9,5 \\
\hline & Questionários acerca da qualidade ... & 2 & 9,5 \\
\hline & Tempo de atendimento do pedido ... & 1 & 4,7 \\
\hline & Outro & 0 & 0,0 \\
\hline \multirow{2}{*}{$\begin{array}{l}\text { Existe sistema de avaliação e cor- } \\
\text { reção de falhas }\end{array}$} & Sim & 8 & 38,0 \\
\hline & Não & 13 & 62,0 \\
\hline
\end{tabular}

Fonte: Elaboração própria (2020). 
Fica evidenciado que $90,4 \%$ dos hotéis realizam pesquisa do nível de satisfação dos hóspedes, fazendo-o diariamente, através do indicador do Booking (71,4\%), 9,5\% disponibilizam questionários que são respondidos pelos hóspedes. Destaca-se que 38,0\% dos hotéis fazem uso de um sistema de avaliação e correção de falhas. Esses sistemas de avaliação, de acordo com os achados de Atkinson et al. (2000, p.94), levam ao controle dos métodos e instrumentos que os membros da empresa usam para mantê-la em acordo com os planos traçados, a fim de atingir os objetivos propostos.

\subsection{A importância da medição de desempenho na tomada de decisões das empresas hoteleiras, indicadores utili- zados e finalidades dos indicadores}

Na Tabela 7 constata-se a importância dada à utilização da medição do desempenho com vista à tomada de decisões. Os 100\% dos hotéis fazem reuniões para debater o desempenho do hotel, só que 33,3\% dos hotéis realizam estas reuniões de forma mensal. Identifica-se que $90,4 \%$ dos hotéis comparam os resultados obtidos com os planejados. Crepaldi (2008, p.2) corrobora com esses achados quando afirma que "as empresas estão em constantes mudanças e cada vez mais necessitam de controles precisos e de informações oportunas sobre seu negócio para adequar suas operações às novas situações de mercado". Os motivos que mais se destacaram entre os hotéis para a medição do desempenho foram: o estabelecimento de metas para melhorias, revisão e alteração de procedimentos da empresa, redução de custos e a melhoria da qualidade.

\begin{tabular}{|c|c|c|c|}
\hline Variável & Categoria & $\mathbf{N}$ & $\%$ \\
\hline \multirow{8}{*}{$\begin{array}{l}\text { Existem reuniões para o debate } \\
\text { sobre desempenho do hotel } \\
\text { Com que frequência estas reuni- } \\
\text { ões acontecem }\end{array}$} & Sim & 21 & 100,0 \\
\hline & Não & 0 & 0,0 \\
\hline & Mensalmente & 7 & 33,3 \\
\hline & Bimestralmente & 2 & 9,5 \\
\hline & Trimestralmente & 3 & 14,2 \\
\hline & Semestralmente & 5 & 23,0 \\
\hline & Anualmente & 3 & 14,2 \\
\hline & Outro (semanalmente, quinzenalmente) & 1 & 5,0 \\
\hline \multirow{9}{*}{$\begin{array}{l}\text { Compara resultados obtidos com } \\
\text { os planejados } \\
\text { A medição de desempenho pode } \\
\text { trazer para o hotel }\end{array}$} & Sim & 19 & 90,4 \\
\hline & Não & 2 & 9,5 \\
\hline & $\begin{array}{l}\text { Comparação do seu desempenho com o de outras em- } \\
\text { presas }\end{array}$ & 5 & 23,8 \\
\hline & Estabelecimento de metas para melhoria & 18 & 85,7 \\
\hline & Marketing da empresa & 3 & 14,2 \\
\hline & Revisão e alteração de procedimentos da empresa & 17 & 80,9 \\
\hline & Redução de custos & 12 & 57,1 \\
\hline & Motivação para melhoria da qualidade & 8 & 38,0 \\
\hline & Outros & 2 & 9,5 \\
\hline
\end{tabular}

Fonte: Elaboração própria (2020).

A Tabela 8 apresenta os indicadores de desempenho financeiro utilizados pelos hotéis. Observou-se que os indicadores mais utilizados, com percentual acima de 80\%, foram: Receita total e Faturamento (ambos com 85,7\%), seguido da Margem de lucro com 71,4\%. O indicador participação do cliente no faturamento foi o menos utilizado com destaque de $38,0 \%$.

\begin{tabular}{lrl} 
Tabela 8 - Indicadores de desempenho financeiros utilizados pelo hotel & & \\
\hline \multicolumn{1}{c}{ Indicadores financeiros utilizados pelo Hotel } & N & $\%$ \\
\hline Rentabilidade & 16 & 76,1 \\
Receita Total & 18 & 85,7 \\
Margem de Lucro & 15 & 71,4 \\
Faturamento - Valor & 18 & 85,7 \\
Participação do cliente no Faturamento & 8 & 38,0 \\
Evolução dos custos & 9 & 42,8 \\
\hline
\end{tabular}

Fonte: Elaboração própria (2020).

Na Tabela 9 destaca-se a utilização dos indicadores de desempenho não financeiros (na perspectiva do cliente). Destaca-se aqui que os indicadores satisfação dos clientes $(95,2 \%)$ e fidelidade dos clientes com $80,9 \%$ são os 
mais utilizados, seguidos pela evolução na posição do mercado com 71,4\% e o indicador participação no mercado com $66,6 \%$.

\begin{tabular}{lrr} 
abela 9 - Indicadores de desempenho não financeiros (na perspectiva do cliente) & utilizados pelo hotel \\
\hline Indicadores não financeiros (na perspectiva do cliente) utilizados pelo Hotel & $\mathrm{N}$ & $\%$ \\
\hline Fidelidade dos clientes & 17 & 80,9 \\
Evolução no n ${ }^{\circ}$ de clientes & 15 & 71,4 \\
Satisfação dos clientes & 20 & 95,2 \\
Participação no mercado & 14 & 66,6 \\
Evolução na posição do mercado & 15 & 71,4 \\
Tempo de permanência no hotel & 13 & 61,9 \\
Número de reclamações & 12 & 57,1 \\
Perfil do consumidor & 4 & 19,0 \\
\hline
\end{tabular}

Fonte: Elaboração própria (2020).

Na Tabela 10, evidenciam-se os indicadores não financeiros (processos internos) e verifica-se que os indicadores mais utilizados foram o de Taxa média de ocupação $(90,4 \%)$ e reclamações de clientes $(76,1 \%)$, seguidos dos indicadores tempo de atendimento do pedido do cliente (52,3\%) e evolução do número de funcionários (42,8\%). Índice de atendimento completo de pedidos e $\mathrm{N}^{\circ}$ de funcionários com contrato a prazo (ambos com 14,2\%) são os indicadores que se apresentam como os menos utilizados.

Tabela 10 - Indicadores de desempenho não financeiros (processos internos) utilizados pelo hote

\begin{tabular}{lrc}
\multicolumn{1}{c}{ Indicadores não financeiros (processos internos) utilizados pelo Hotel } & $\mathrm{N}$ & $\%$ \\
\hline Taxa média de ocupação & 19 & 90,4 \\
Tempo de atendimento do pedido do cliente & 11 & 52,3 \\
Tempos para solucionar as reclamações & 8 & 38,0 \\
Índice de atendimento completo de pedidos & 3 & 14,2 \\
Evolução do n ${ }^{\circ}$ de funcionários & 9 & 42,8 \\
$N^{\circ}$ de funcionários com contrato a prazo & 3 & 14,2 \\
$N^{\circ}$ Fornecedores & 4 & 19,0 \\
Reclamações de clientes & 16 & 76,1 \\
\hline
\end{tabular}

Fonte: Elaboração própria (2020).

Na Tabela 11, constata-se que o indicador mais utilizado é qualidade do serviço (80,9\%) seguido do indicador satisfação dos trabalhadores (38,0\%). Para Banker, Poter e Srinivasan (2000), os indicadores não financeiros, aqui relatados, são mais eficazes para o futuro que os financeiros, contudo, auxilia os administradores nas ações a longo prazo. Destacam-se ainda os indicadores Investimento em ação social e Indicadores ambientais como os menos utilizados (ambos com 9,5\%).

\begin{tabular}{lrr}
\multicolumn{2}{l}{ Tabela 11 - Indicadores de desempenho não financeiros (aprendizado e crescimento) utilizados pelo hotel } \\
\hline \multicolumn{1}{l}{ Indicadores não financeiros (aprendizado e crescimento) utilizados pelo Hotel } & $\mathbf{N}$ \\
\hline Satisfação dos trabalhadores & 8 & 38,0 \\
Investimento em ação social & 2 & 9,5 \\
Investimento em treinamento & 4 & 19,0 \\
Investimento em atendimento & 5 & 23,8 \\
Investimento em tecnologia & 6 & 28,5 \\
Investimento em equipamentos & 7 & 33,3 \\
Qualidade do serviço & 17 & 80,9 \\
Indicadores ambientais & 2 & 9,5 \\
\hline
\end{tabular}

Fonte: Elaboração própria (2020).

A Tabela 12 apresenta a percepção dos gestores dos hotéis quanto ao uso dos indicadores, e a maioria dos gestores afirma que a grande finalidade dos indicadores é a gestão operacional (80,9\%), seguido do objetivo de atingir os resultados pretendidos $(76,1 \%)$ e do objetivo de rever a política estratégica da empresa $(52,0 \%)$. 
Tabela 12 - Finalidades do uso dos indicadores

\begin{tabular}{lrc}
\multicolumn{1}{c}{ Finalidades } & N & $\%$ \\
\hline Gestão operacional & 17 & 80,9 \\
Gestão orçamental & 7 & 33,3 \\
Rever a política estratégica da empresa & 11 & 52,0 \\
Comunicar a missão e os objetivos do hotel & 4 & 20,5 \\
Atingir os resultados pretendidos & 16 & 76,1 \\
\hline
\end{tabular}

Fonte: Elaboração própria (2020).

\subsection{Utilização de indicadores na perspectiva do BSC}

A Tabela 13 objetiva, de acordo com a estrutura do BSC (financeira, clientes, processos internos e aprendizagem e crescimento), destacar os indicadores de desempenho utilizados pelo hotel. Atribuiu-se os pesos 1, 3 e 5, respectivamente Pouco importante, Importante e Muito importante, sobre os indicadores financeiros (medidas quantitativas, expressas em valor monetário) e não financeiros (incluem indicadores da perspectiva clientes, processos internos, aprendizagem e crescimento). Constata-se que dos indicadores financeiros utilizados, os mais importantes são: faturamento-valor, rentabilidade, margem de lucro e receita total. Com relação aos indicadores não financeiros, destacam-se: participação no mercado, qualidade do serviço, tempo de permanência no hotel, reclamações dos clientes, satisfação do cliente, fidelidade dos clientes, número de reclamações, evolução na posição de mercado e taxa média de ocupação. Esses achados vêm corroborar com Merchant (2006), quando afirma que a construção/utilização de indicadores de desempenho de forma adequada está diretamente relacionada ao sucesso de um sistema de avaliação de desempenho organizacional.

\begin{tabular}{lcll}
\multicolumn{2}{l}{ Tabela 13 - Pesos atribuídos aos indicadores de desempenho financeiro e não financeiros } & Peso \\
\hline Indicadores não financeiros & Peso & Indicadores financeiros & 5 \\
\hline Número de fornecedores & 1 & Faturamento - valor & 3 \\
Evolução do número de funcionários & 3 & Participação do cliente no faturamento & 5 \\
Investimento em ação social & 1 & Rentabilidade & 5 \\
Investimento em treinamento & 3 & Margem de lucro & 3 \\
Participação no mercado & 5 & Evolução dos custos & 5 \\
Qualidade do serviço & 5 & Receita total & \\
Tempo de atendimento do pedido do cliente & 3 & & \\
Tempo de permanência no hotel & 5 & \\
Reclamações dos clientes & 5 & \\
Satisfação do cliente & 5 & \\
Fidelidade dos clientes & 5 & \\
Número de reclamações & 5 & \\
Satisfação dos trabalhadores & 3 & \\
Evolução na posição de mercado & 5 & \\
Indice de atendimento completo de pedidos & 1 & \\
Perfil do consumidor & 1 & \\
Tempo para solucionar as reclamações & 3 \\
Investimento em atendimento & 1 \\
Investimento em equipamento & 3 \\
Número de funcionários com contrato a prazo & 1 \\
Evolução do número de clientes & 5 \\
Investimento em tecnologia & 3 \\
Taxa média de ocupação & 5 \\
Indicadores ambientais & 1 & \\
\hline
\end{tabular}

Fonte: Elaboração própria (2020).

Portanto, constata-se que os hotéis dão mais importância aos indicadores financeiros do que aos não financeiros (3 a 5, quase sempre 5), enquanto que nos indicadores não financeiros, os mais importantes, são os que induzem resultados a curto prazo (como, por exemplo, a fidelidade de clientes), enquanto os que induzem resultados a longo prazo como a formação dos trabalhadores e o investimento em tecnologia só atingem um valor médio (3).

\section{CONSIDERAÇÕES FINAIS}

Os gestores hoteleiros devem estar atentos as ferramentas de gestão que possibilitam enfrentar os desafios e as oportunidades que o desenvolvimento do turismo traz ao setor. Nesse sentido, este estudo buscou conhecer os indicadores de desempenho utilizados pelos empreendimentos hoteleiros da cidade de Braga/PT, identificando 
como são utilizados, à luz da teoria dos indicadores do Balanced Scorecard desenvolvidos por Kaplan e Norton (1997).

Em relação ao perfil dos gestores dos hotéis de Braga, constatou-se que é majoritariamente do gênero masculino e que a maioria é formado nas áreas de Gestão, Economia e Turismo, sendo grande parte destes gerentes e/ou proprietários. Além disso, 57\% dos hotéis são classificados nas categorias 2 ou 3 estrelas e quase $67 \%$ enquadramse no tipo de hospedagem de lazer, descanso e negócio.

É importante destacar que esses estabelecimentos que se encontram no mercado, em média, há 25 anos, são de pequeno porte, com aproximadamente 30 Unidades Habitacionais e média de 11 colaboradores e, sobretudo, que $57 \%$ dos estabelecimentos não utilizam qualquer tipo de financiamento bancário.

No que se refere à gestão, o estudo evidenciou que $81 \%$ dos hotéis utilizam Sistema de Medição de Desempenho (SMD) e que parte destes $(56,1 \%)$ faz a utilização de um sistema de desempenho com indicadores calculados através do EXCEL. Em todas as empresas hoteleiras pesquisadas são realizadas reuniões com o objetivo de debater sobre o desempenho e a maioria compara os resultados com o que foi planejado.

De acordo com os dados, dentre os motivos que mais se destacaram para a medição de desempenho nos hotéis estão o estabelecimento de metas para melhorias, revisão e alteração de procedimentos da empresa, redução de custos e a melhoria da qualidade. Os indicadores mais utilizados são os financeiros (rentabilidade, receita total e faturamento - valor) e nos indicadores não financeiros, os mais utilizados são os de satisfação dos clientes e fidelidade aos clientes e, segundo a literatura, este último induz a resultados a curto prazo.

A falta de investimento em estudo dos dados e melhorias nas áreas não financeiras de longo prazo, como treinamento, investimento em tecnologia, equipamentos e fornecedores, podem ser um revés no momento atual global, onde são impostas normas de trabalho bem rígidas para o combate à disseminação da Covid-19. Se a empresa tem essas áreas bem trabalhadas, usando o Brasil como exemplo, adotar as políticas internas de implementação de recomendações, como as feitas pelo Ministério do Turismo para o recebimento do "Selo: Turismo responsável", pode acontecer de forma fluida e sem muitos problemas. Ao final do estudo conclui-se que os gestores dos hotéis se utilizam de ferramentas que corroboram na gestão com efeitos de curto prazo, e que, segundo a literatura, os indicadores não financeiros podem ser mais eficazes para o futuro do que os financeiros, auxiliando os administradores a focarem suas ações nas perspectivas a longo prazo.

Como sugestão para trabalhos futuros, recomenda-se a expansão do universo da pesquisa, abrangendo hotéis de outras regiões de Portugal, para fins comparativos e, dessa forma, obter-se maior generalização dos resultados. Podem ser realizados também estudos sobre como os meios de hospedagem estão se adequando ao cenário imposto pela pandemia.

\section{REFERÊNCIAS}

Amato, Gian. (2021). Portugal começa emitir certificado digital covid-19 e turismo volta ao simples e seguro. Recuperado de https://blogs.oglobo.globo.com/portugal-giro/post/portugal-comeca-emitir-certificado-digitalcovid-19-e-turismo-volta-ao-simples-e-seguro.html

Atkinson, A. A.; Banker, R. D.; Kaplan, R. S., \& Young, S. M. (2000). Contabilidade gerencial (1a ed). São Paulo: Atlas

Badaró, R. A. L. (Org.). (2010). Hotelaria à luz do Direito do Turismo. São Paulo: Senac

Banker, R. D.; Potter, G., \& Srinivasan, D. (2000). Na empirical investigation of na incentive plant that includes nonfinancial performance measures. The accounting Review, 75(1), p. 65-92. https://doi.org/10.2308/accr.2000.75.1.65

Barbosa, A. S. (2010). Uso de indicadores de desempenho nas empresas construtoras brasileiras: diagnóstico e orientações para utilização. (Tese de Doutorado). Curso de Engenharia De Produção, Universidade De São Carlos, São Carlos, SP, Brasil.

Barros, P. C., \& Badiz, M. P. (2005). Prestação de serviços: hotelaria. In: IX Encontro Latino Americano de Iniciação Científica e V Encontro Latino Americano de Pós-Graduação - Universidade do Vale do Paraíba, 2005, São José dos Campos. Anais eletrônicos, 2005. Recuperado de: https://biblioteca.univap.br/dados/INIC/cd/inic/IC6\%20anais/IC6-116.pdf

Bisbe, J., \& Barrubés, J. (2012). The balanced scorecard as a management tool for assessing and monitoring strategy implementation in health care organizations. Rev Esp Cardiol. 65 (10), p. 919-927. 
Bortoluzzi, S. C. Ensslin, S. R., \& Enssilin, L. (2010). Avaliação de desempenho dos aspectos tangíveis e intangíveis da área de mercado: estudo de caso em uma média empresa industrial. RBGN Revista Brasileira de Gestão de Negócios, 12(37), p. 425-446.

Braam, G., \& Nijssen, E. (2011). Exploring antecedents of experimentation and implementation of the balanced scorecard. Journal of Management and Organization. Cambridge, 17(6), p. 714-728. https://doi.org/10.5172/imo.2011.714

Callado; A. L. C; Callado, A. A. C., \& Almeida, M. A. (2008). Determinando Padrões de Utilização de Indicadores de Desempenho: Um Estudo em Micro e Pequenas Empresas da Cidade de João Pessoa. Revista UnB Contábil, 11(1-2), p. 18-29). https://doi.org/10.47179/abcustos.v3i2.45

Castelli, G. (1999). Administração Hoteleira. Caxias Do Sul: Educs.

Crepaldi, S. A. (2008). Contabilidade Gerencial: Teoria e Prática. (4a ed.) Atlas.

Crispim, S., \& Lugoboni, L. (2012). Avaliação de desempenho organizacional: Análise comparativa dos modelos teóricos e pesquisa de aplicação nas Instituições de Ensino Superior da Região Metropolitana de São Paulo. Revista Portuguesa e Brasileira de Gestão 11 (1), p. 41-54

Desporto. (2018). Braga recebe distinção de Melhor Cidade Europeia do Desporto 2018. Recuperado de https://desporto.sapo.pt/geral/artigos/braga-recebe-distincao-de-melhor-cidade-europeia-do-desporto$\underline{2018}$

Faria, R. T. de. (2007). A Gestão Estratégica com o uso do Balanced Scorecard como diferencial competitivo no setor supermercadista (Trabalho de conclusão de curso). Universidade Federal de Juiz de Fora - UFJF, Minas Gerais, MG, Brasil.

Frigo, M., \& Krumwiede, K.R. (2000). The balanced scorecard: a winning performance measurement system. Strategic Finance, 81(7), p. 50-4.

Lei $n^{\circ} 11.771$ de 17 de setembro de 2008 - Dispõe sobre a Política Nacional de Turismo. Recuperado de http://www.planalto.gov.br/ccivil 03/ Ato2007-2010/2008/Lei/L11771.htm

Gomes, M. (2018). Passaram por Braga mais de um milhão de turistas entre 2013 e 2018. In: Semanário v. Recuperado de https://semanariov.pt/2018/09/27/passaram-por-braga-mais-de-um-milhao-de-turistas-entre2013-e-2018

Greiling, D. (2010). Balanced scorecard implementation in german non-profit organizations. International Journal of Productivity and Performance Management. Bradford, 59 (6), p. 534-554. https://doi.org/10.1108/17410401011063939

Gullo, M. C. R. (2020). A economia na pandemia Covid-19: algumas considerações. Rosa dos Ventos - Turismo e Hospitalidade, 12 (3a ed. especial Covid 19, 1-8). http://doi.org/10.18226/21789061.v12i3a05

Haktanir, M., \& E Harris, P. (2005). Performance Measurement Practice in an Independent Hotel Context. International Journal of Contemporary Hospitality Management, $17 \quad$ (1), p. $39-50$ https://doi.org/10.1108/09596110510577662

Hronec S. M. (1994). Sinais Vitais: usando medidas de desempenho da qualidade, tempo e custo para traçar a rota para o futuro de sua empresa. Makron Books

Hendricks, K.; Menor L., \& Wiedman C. (2012). Adoption of the balanced scorecard: a contingency variables analysis. Canadian Journal of Administrative Sciences, Chichester, 29 (2), p. $124-138$ https://doi.org/10.1002/cjas.229

Hoffecker, J., \& Goldenberg, C. (1994). Using the balanced scorecard to develop company-wide performance measures. Journal of Cost Management, 8(3), p. 5-17.

INE (2020). Matrizes Simétricas Input-Output. Lisboa, Portugal. Recuperado de https://www.ine.pt/xportal/xmain?xpid=INE\&xpgid=ine destaques\&DESTAQUESdest boui=4 modo $=2 \& x$ lang $=p t$

Kaplan, R.S., \& Norton, D.P. (1992). The balanced scorecard - measures that drive performance. Harvard Business Review, January/February, pp. 1-79 https://doi.org/10.2307/41165876

Kaplan, R.S., \& Norton, D.P. (1993). Putting the balanced scorecard to work. Harvard Business Review, September/October, pp. 134-47

Kaplan, R.S., \& Norton, D.P. (1996a). Using the balanced scorecard as a strategic management system. Harvard Business Review, 74, pp. 75-85. 
Kaplan, R.S., \& Norton, D.P. (1996b). Linking the balanced scorecard to strategy. California Management Review, 39(1), p. 53-79.

Kaplan, R.S., \& Norton, D.P. (1996c). The Balanced Scorecard. Harvard Business School Press, Boston, MA, EUA.

Kaplan, R. S., \& Norton, D. P. (1997). A estratégia em ação: Balanced Scorecard. (6a ed.). Rio de Janeiro: Campus

Kaplan, R.S., \& Norton, D.P. (2001). The Strategy-Focused Organization. Harvard Business School Press, Boston, MA, EUA. https://doi.org/10.1108/sl.2001.26129cab.002

Kiyan, F. M. (2001). Proposta para desenvolvimento de indicadores de desempenho como suporte estratégico. (Dissertação). Escola de Engenharia da USP.

Kollberg, B., \& ELG, M. (2001). The practice of the balanced scorecard in health care services. International Journal of Productivity and Performance, Bradford $60 \quad$ (5), p. 427-445) https://doi.org/10.1108/17410401111140374

Kutzman, J. (1997). Is your company off course? Now you can find out why. Fortune, 135 (3), p. 128-30.

Lebas, M., \& Euske, K. (2004). A conceptual and operational delineation of performance. In: A. Neely (Ed.), Business performance measurement (pp. 65-79). Cambridge: Cambridge University Press, 2002 https://doi.org/10.1017/CBO9780511753695.006

Leite, D. C. C. (2004). Investigação sobre a medição de desempenho em pequenas empresas hoteleiras do nordeste brasileiro. (Dissertação de Mestrado). Curso de Ciências Contábeis, UNB/UFPB/UFPE/UFRN, João Pessoa, PB, Brasil.

Lingle, J.H., \& Schiemann, W.A. (1996). From balanced scorecard to strategic gauges: is measurement worth it? Management Review, 5(3), p. 56-61.

Lugoboni, L. F.; Fontes F. de S., \& Andrade D. A. da C. (2013). Avaliação de Desempenho Organizacional: medição de desempenho em hotéis do estado de São Paulo. In: X Seminário da Associação Nacional Pesquisa e Pós-Graduação em Turismo. Universidade de Caxias do Sul - UCS, Caxias do Sul, RS, Brasil.

Lunkes, R. J. (2004). Manual de Contabilidade Hoteleira. Atlas

Machado, M. J. C. V. (2013). Balanced scorecard: an empirical study of small and medium size enterprises. RBGN Revista Brasileira de Gestão de Negócios, São Paulo, 15(46), p. 129-148. https://doi.org/10.7819/rbgn.v15i46.1175

Maisel, L.S. (2001). Performance Measurement Practices Survey. American Institute of Public Accountants, New York, NY, EUA.

Martins, L. A. V., \& Flores, L. C. S. (2017). Comportamento Estratégico: Perfil dos Gestores Hoteleiros da Região do Vale do Itajaí/Santa Catarina. Revista Hospitalidade, 14 (2), 01-23 https://doi.org/10.21714/2179$\underline{9164.2017 v 14 n 2.764}$

Merchant, K. A. (2006). Measuring general manager's performances: Market, accounting and combination of measures systems. Accouting, Auditing \& Accountability Journal, 19 (6), p. 893-917. https://doi.org/10.1108/09513570610709917

Mota, K. C. N. (2001). Marketing Turístico: Promovendo uma Atividade Sazonal. São Paulo: Atlas

Mundo Lusiada. (2019). Bom Jesus de Braga e Palácio de Mafra classificados patrimônio mundial da UNESCO. Recuperado de https://www.mundolusiada.com.br/box1/bom-jesus-de-braga-e-palacio-de-mafra-classificados-patrimonio-mundial-da-unesco/

Nascimento, D. J.; Pederneiras, M. M. M.; Lopes, J. E. G., \& Sousa, C. P. (2015). Um Estudo Acerca da Relação Entre os Gestores de Micro e Pequenas Empresas do Ramo de Supermercados e a Contabilidade Gerencial: algumas evidências. In: I Seminário de Ciências Contábeis e Atuariais da UFPB, 2015, João Pessoa-PB. Anais. Recuperado de http://www.media.wix.com/ugd/ee51b6 fe19f6d2f4f44edca 228607d757edaa4.pdf

Neely, A. (1998). Measuring business performance. London: The Economics

Organização Mundial do Turismo - OMT. (2019). World Tourism Barometer 2019. Recuperado de https://webunwto.s3.eu-west-1.amazonaws.com/s3fs-public/2020-01/UNWTO_Barom20_01_January_excerpt 0.pdf

Organização Mundial do Turismo - OMT. (2021). UNWTO World Tourism Barometer and Statistical Annex, March 2021. https://doi.org/10.18111/wtobarometereng.2021.19.1.2 
Paiva, C. P.; Magalhães Junior, D. C.; Leandro, M. R. P.; Garcia, T. Z.; Vendrame,M. de C. R., \& Lima, A.B. (2010). A evolução da qualidade nos ciclos de serviços hoteleiros: um estudo de caso no Cristal Palace Hotel. Revista Científica do Unisalesiano - Lins - SP, 1(2)

Paixão, W.B.; Cordeiro, I.J.D., \& Leite, N.K. (2021). Efeitos da pandemia do COVID- 19 sobre o turismo em Fernando de Noronha ao longo do primeiro semestre de 2020. Revista Brasileira de Pesquisa em Turismo, São Paulo, 1 5(1), 2128. https://doi.org/10.7784/rbtur.v15i1.2128

Petrocchi, M. (2003). Hotelaria: Planejamento e Gestão. São Paulo: Futura

Portaria Regulamentar. Recuperado de https://dre.pt/application/file/70869184

Popp, E. V.; Silva, V. C.; Marques, J.A.; Cardone, R., \& Fernandes, R. (2007). Hotelaria e hospitalidade. São Paulo: IPSIS

Quesado, P. R., \& Rodrigues, L. L. (2009). Factores determinantes na implementação do Balanced Scorecard em Portugal. Revista Universo Contábil, ISSN 1809-3337, FURB, Blumenau, 5 (4), p. 94-115, out./dez. https://doi.org/10.4270/ruc.2009433

Rodrigues, L. H; Schuch, C., \& Pantaleão, L. H. (2003). Uma abordagem para construção de sistemas de indicadores alinhados a teoria das restrições e o Balanced Scorecard. In: Encontro da Associação Nacional de Programas de Pós-Graduação em Administração, 27, 2003, Atibaia. Anais. Atibaia: ANPAD

Travelbi, (2018). Turismo na Europa. Recuperado de https//travelbi.turismodeportugal.pt/ptpt/Paginas/european-tourism-trends-prospects-quaterly-report-q1-2018.aspx.

\section{Informações dos Autores}

\section{Marcleide Maria Macêdo Pederneiras}

Professora da Universidade Federal da Paraíba/Departamento de Turismo e Hotelaria, João Pessoa, Paraíba - Brasil e Professora Permanente do Mestrado Profissional em Administração Pública da UFCG - PROFIAP. Pós-Doutorado em Contabilidade, Doutorado em Administração, Mestrado e Graduação em Contabilidade.

Contribuições: Concepção da pesquisa, revisão da literatura, coleta dos dados, análise dos dados e discussão.

E-mail: marcleide@gmail.com

ORCID: https://orcid.org/0000-0001-9092-807X

\section{Robson do Vale Silva}

Graduando na Universidade Federal da Paraíba - João Pessoa, Paraíba - Brasil. Departamento de Turismo e Hotelaria, João Pessoa, Paraíba - Brasil

Contribuições: Concepção da pesquisa, revisão da literatura, coleta dos dados, análise dos dados e discussão.

E-mail: robbye.valley@gmail.com

ORCID: https://orcid.org/0000-0002-8084-3512

\section{Paula Dutra Leão de Menezes}

Professora do Departamento de Turismo e Hotelaria (DTH) - CCTA -UFPB - João Pessoa - PB. Doutora em Humanidades y Artes (Educação) pela Universidad Nacional de Rosario (UNR).

Contribuições: Revisão da literatura, análise de dados e discussão dos resultados.

E-mail: leaopaula@hotmail.com

ORCID: http://orcid.org/0000-0001-9736-8802

\section{Jonathan Muniz Soares}

Bacharel em Hotelaria pela Universidade Federal da Paraíba, João Pessoa, PB, Brasil. Departamento de Turismo e Hotelaria, João Pessoa, Paraíba - Brasil

Contribuições: Concepção da pesquisa, revisão da literatura, coleta dos dados, análise dos dados e discussão.

E-mail: jms.j|r@gmail.com

ORCID: https://orcid.org/0000-0001-9803-5049 